\title{
CULTURA HACEDOR, MODELIZADOR PARAMETRICO Y PROTOTIPADO DIGITAL. Un caso de prácticas didácticas en entorno post-digital en la carrera de Diseño Industrial
}

\author{
MAKER MOVEMENT, PARAMETRIC MODELING, DIGITAL PROTOTYPE. A case of teaching \\ practices in post-digital environment in the career of Industrial Design
}

\author{
Dra. Arq. Diana Rodríguez Barros \\ FAUD UNMdP, Argentina \\ dibarros@mdp.edu.ar
}

\author{
Dis. Ind. Pablo Pellizzoni \\ FAUD UNMdP, Argentina \\ Dirección de email
}

\begin{abstract}
In post-digital, virtual and interconnected web environments, happen participatory actions and practices related to the concept of Collective Intelligence. Collaborative habits of creation and validation of knowledge are stimulated. Cognitive ecosystems are enabled facilitating participation in egalitarian environments. Are seen as propitius environments for the development of the Culture Maker / Maker Movement redefining innovative relationships between society, culture and technology. In this direction, we present a teaching practice done in the tii1-2 FAUD UNMdP, developed from the perspective of Design Thinking, in design lab mode and digital fabrication FabLab. Therefore, it enabled us to explore this trend that makes it easy to share the what, how and why is created.
\end{abstract}

Keywords: Didactics practices; Industrial Design; Maker Movement; Parametric Modeling; Digital Prototype.

\section{Introducción}

En entornos post-digitales, virtuales e interconectados a la Web, suceden acciones participativas y prácticas relacionados con el concepto de Inteligencia Colectiva (Cobo y Moravec, 2011). Se estimulan hábitos colaborativos de creación y validación del conocimiento. Se habilitan ecosistemas cognitivos facilitando participación en ambientes igualitarios Se observan como ambientes propicios para el desarrollo de un fenómeno particular, la Cultura del Hacedor Maker Movement redefiniendo relaciones innovadoras entre sociedad, cultura y tecnología.

Básicamente, esta expresión cultural reconoce empoderamiento de personas, acceso al conocimiento abierto, aplicaciones libres y originales formas de aprobación entre pares (Acaso, 2016). De forma específica, las innovaciones tecnológicas no son creadas y producidas de forma exclusiva por empresas, grandes fabricantes y compañías multinacionales, sino cada persona o grupo tiene acceso a herramientas y posibilidades para diseñar, rediseñar y producir sus propios productos, sin necesidad de inversiones importantes.

\section{Marco conceptual}

La Cultura del Hacedor resulta de la compleja hibridación de cultura libre, autorreplicabilidad, fabricación digital y paradigma DIY. Tal circunstancias nos conduce a centrarnos en los movimientos de la fabricación personal propios de laboratorios abiertos de fabricación digital, los FabLabs y del Maker Movement.

Como indica Pau Llop (2013), la fabricación digital en tanto práctica abierta de producción entre iguales, produce una disrupción en el modelo productivo industrial tradicional al independizar diseños y medios de producción, facilitando a cualquier persona que en cualquier lugar del mundo integre y participe de estos originales procesos de generación de valor. La aplicación de los principios de la cultura libre a peer production precipita su capacidad transformadora desde inéditos modos de idear diseños de entornos y objetos, crear herramientas, y alterar procesos de producción y consumo.

El argumento fundante del movimiento Maker es aprender desde y a partir de la experiencia. Su originario referente ha sido DIY (Do It Yourself, Házlo tú mismo), pero ha ido mutando hacia DIWO (Do It With Others, Házlo con otros), desde abordajes transdiciplinares y tanto combinando computación, diseño, robótica, entre otras disciplinas; como estimulando el trabajo colectivo, la generación de conocimiento en comunidad y la invalidación del individualismo.

Para Arango Sarmiento (2016), una serie de principios simples y concretos desencadenan acciones que orientan esta movida cultural. En un orden no secuencial, reconoce, Crear y co-crear, como paso del idear al hacer, principio 
fundamental a través de unirse a otros, integrarse a redes, validarse entre pares y generar son las principales consignas; Aprender, auto-aprender y experimentar, como actualización contante desde procesos de educación formal e informal en diseño de aplicaciones, programación o inclusive programación básica, trabajo y exploración; Compartir, como la generación de conocimiento en comunidad que tiene sentido cuando es participado, intervenido, retroalimentado, modificado y replicado por otros; Generar herramientas, como modo de acceder a nuevas recursos adecuados y dejarlos a disposición de otros para que puedan usarlos; Jugar, como desencadenante principal de la innovación desde posiciones lúdicas; Participar y apoyar, como modalidad para intercambiar avances, desarrollos, experiencias desde redes y encuentros; Permutar, como actitud de fluidez y transformación constante para empoderarse de la transformación y ser parte activa en la innovación.

De tal forma, la Cultura del Hacedor Maker Movement, se expande como sub-cultura contemporánea, englobando prácticas y relaciones resultantes de la fusión de modos particulares de fabricación personal distribuida fabbing alejados de la industria a gran escala, y necesariamente con acompañamientos de desarrollos paralelos de acceso abierto a hardware de fabricación digital y de propagación de información específica sobre uso y diseños desde la Web.

\section{Presentación del caso}

En esta dirección, presentamos una práctica didáctica realizada durante el $2^{\circ}$ cuatrimestre del ciclo lectivo 2015 , en el Taller de Informática Industrial nivel 2, con estudiantes de la orientación Producto del $3^{\circ}$ año de la carrera de Diseño Industrial de la Facultad de Arquitectura, Urbanismo, Diseño, Universidad Nacional de Mar del Plata, Argentina. Fue desarrollada desde la perspectiva del Pensamiento de Diseño, uno de los abordajes contemporáneos validados para identificar acciones creativas, co-creativas e innovadoras (Brown, 2016). Tal postura implica realizar enfoques inter y multidisciplinarios, valorar la pseudoserendipia, expresar un tipo de pensamiento integrador de naturaleza abductivo, relacionar pensamiento deductivo y fiable propio de las ciencias abstractas junto a un pensamiento inductivo y válido propio de las ciencias experimentales, recurrir a la experimentación, así como verificación, visualización, y comunicar según instancias de prototipado rápido (Freire, 2012).

La experiencia se enfocó en intervenciones de recreación y rediseños de productos, a la manera de accesorios rémoras, a ser utilizados por los propios estudiantes en sus actividades cotidianas en la carrera, con posibilidades reales de fabricación con mínimas inversiones. Los estudiantes abordaron el rediseño de maquinarias simples, que facilitaran tareas complementarias en la confección de prototipos exigidos en el transcurso de la carrera. Con diversos mecanismos en desuso y posterior reciclados, según las necesidades, se dedicaron a resolver el diseño y rediseño de las envolventes y producir los prototipos correspondientes. En la experiencia, curricularmente orientada hacia la indagación y el aprendizaje de programas de computación gráfica aplicada la diseño, se recurrió a aplicaciones CAD 3D paramétricas para modelado mecánico factibles de modelar piezas y conjuntos, así como recuperar documentación técnica y otro tipo de información necesaria para la producción. Se experimentó luego con diversas tecnologías de fabricación digital (impresión 3D, fresado y corte láser entre otras) según requerimientos de las maquinarias diseñadas. Finalmente se realizó una ponderación de pares entre los mismos estudiantes, verificando condiciones de usabilidad eficaz y eficientes de los productos generados, junto a experiencias de usuario satisfactorias.

Nos interesó, desde nuestros estudios y prácticas docentes realizadas, conformar un ámbito de trabajo que incorporara incursiones en modalidad FabLab. De tal forma, nos habilitó para explorar esta tendencia que facilita la posibilidad de compartir el qué, el cómo y el por qué se crea.

Desde este encuadre, afrontamos la creación y la cocreación, así como la puesta en marcha procesos de transformación e innovación, reconociendo tres procesos básicos. Primero, la exploración de nuevas ideas u oportunidades que van a desencadenar actividades de descubrir y formular; segundo, el desarrollo de modelos y prototipos para conceptualizar y detallar; tercero, la implementación junto a la respectiva evaluación del producto.

\section{Metodología y técnicas empleadas}

La experiencia se desarrolló en modalidad Taller de Diseño, en ambiente de estudio sustentadas por la acción, la conceptualización y la reflexión. Hemos recurrido al aprendizaje y al uso de aplicaciones estándares en entornos conectados a la Web, simulando prácticas disciplinares que desarrollan y verifican formas flexibles de construcción de conocimiento. En términos amplios, realizamos la experiencia a manera laboratorios locales donde desarrollar intervenciones de diseño y acceder a las exploración de instancias de fabricación digital.

Metodológicamente, retomando y reformulando experiencias docentes anteriores (Rodríguez Barros 2014; 2013), adscribimos a la perspectiva del Pensamiento de Diseño. Se participó entonces en espacios de acción iniciados en espacios para Inspirar, continuando con espacios para Idear, para Experimentar, para Implementar y para Prototipar (Brown, 2016 op.cit.). Nos orientamos según tácticas explícitas propias de tales espacios de acción, desde etapas de complejidad creciente, no estrictamente secuenciales, y con las necesarias retroalimentaciones y ajustes.

La secuencia de acciones recorrió a manera orientadora una serie de fases. Se inició a partir de disparadores formalizados desde narrativas previas, sobre experiencias personales de los estudiantes y la consecuente reformulación crítica para tornarlas en experiencias gratificantes, se planteó el eje estructurante de la práctica. La misma se ha enfocado en el concepto maquinaria complemento, en tanto integrante de familia de productos y en el diseño de maquinarias, para asistir acciones a los 
propios estudiantes en sus actividades cotidianas en la carrera (accesorios y rémoras para la producción de maquetas) con posibilidades factibles de fabricación digital y con costos mínimos.

Luego precisaron perfil de usuario, contexto uso y modalidad de uso. Indagaron sobre objetos asimilables. Seleccionaron los condicionantes de la definición estratégica y de concepto del complemento/ accesorio - rémora a diseñar.

Realizaron el relevamiento y el registro de objetos asimilables para su posterior intervención analizando morfología, estructura organizativa, ergonomía, dimensiones, usos, materialidad, etc.

Diseñaron el accesorio que debería viabilizar la experiencia de usuario gratificante inicialmente propuesta, este objeto nuevo (podía ser más de uno) debía estar en línea con el objeto inicial y con diferente función específica compartiendo un mismo ámbito de uso; modelaron el accesorio - rémora tridimensionalmente, indagando diferentes opciones realistas según disponibilidades de aplicaciones NURBs, superficies malladas y modelado paramétrico, respetando dimensiones, morfología, vinculación entre partes, ensamblado, etc.; renderizaron el accesorio rémora con aplicaciones avanzadas para optimizar el grado de rrealismo; generaron videos de animación del modelo 3D.

Fabricaron un prototipado rápido del accesorio - rémora según tecnologías compatibles del tipo de corte láser, tecnologías sustractivas de fresado CNC 2D y 3D (mediante desbaste empleando cartón, espumas, acrílicos o placas de MDF según los casos), y/o tecnologías aditivas de impresión 3D. Produjeron documentación 2D según normativas estandarizada. Asistidos por charlas de expertos y visitas a sitios especializados, exploraron los diferentes opciones disponibles en un FabLab (cortadora láser para ensamblar estructuras 3D desde partes 2D, fresadoras, cortadoras de vinilo, impresoras 3D, entre otras).

Generaron piezas comunicacionales en modalidad infografía y presentación digital interconectada.

Al seguimiento presencial en clase, se sumó la actividad de críticas y correcciones realizada desde un grupo cerrado de Facebook. Asimismo y en forma presencial, se efectuaron intercambios grupales, evaluaciones parciales y finales a manera de enchinchadas, instancias de autoevaluación, instancias de evaluación entre pares a manera de testeo (Ver Figuras 1, 2, 3, 4, 5, 6)

\section{Resultados}

Los productos rediseñados, prototipados y probados han cubierto un espectro amplio. Registramos maquinarias accesorios - rémora del tipo dobladora de varilla de aluminio de baja escala; máquina para impresión al agua water print semiautomática; acesorio rémora para cutter de corte a $45^{\circ}$ y $90^{\circ}$; lijadora de banda desde rotoagujeradora; masilladora de enduido por gravedad; copiador de corte de Polyfan; cabina de pintado semiautomática de maquetas; entre otros.

Analizamos y evaluamos la experiencia, con resultados mayoritariamente muy positivos, siguiendo el modelo de construcción de significaciones y Diseño Experiencia Usuario que condiciona intención de uso y comportamiento (Diller, Shedroff, Rhea, 2008), según indicadores sobre utilidad, facilidad uso, atractivo estético visual, junto a preferencias placenteras percibidas.

Como resultados de la experiencia, hemos registrado el planteo y la solución de problemas de complejidad media con respuestas novedosas y factibilidad productiva relativamente innovadora. En términos amplios, hemos notado mayoritariamente que la experiencia, que ha sido presentada de manera realista desde un curso específico sobre aprendizaje de aplicaciones de computación gráfica según lo indica la currícula de la carrera, ha superado limitaciones instrumentalistas y ha estimulado la posibilidad de indagar en profundidad el planteo y la solución de problemas con respuestas originales.

Hemos observado que se promovieron habilidades cognitivas de mayor grado y compromiso en los estudiantes. Las mismas han estimulado la participación en la co-creación desde la diversidad de criterios y miradas en las intervenciones; del estímulo de motivaciones, asunción de roles varios, movilidad y readaptación en cada etapa; del diálogo y respeto en el grupo expresado a través de la construcción de ambientes comunes y lenguajes compartidos, especialmente estimulado desde en el grupo de apoyo cerrado en redes sociales.

Asimismo hemos detectado avances positivos, tomando como referencia a indicadores planteados a priori sobre comportamientos deseables en procesos de aprendizaje de los estudiantes (Christensen, Dyer, Gregersen, 2011). Al respecto han estado referidos a la revalorización y reafirmación de comportamientos sobre observación, reconociendo nuevos modos de acción; a la asociación, generando vinculaciones entre temas y supuestamente desvinculados y derivados de ambientes ajenos; al cuestionamiento, provocando y discutiendo modos posibles de acción; a la experimentación, explorando alternativas de aprendizaje permanente para probar, desarmar, ver que hay dentro, prototipar, reflexionar y realimentar el proceso; a la integración a redes, conectándose, escuchando y estableciendo vínculos ante la diversidad.

De tal forma, hemos verificado mayoritariamente facilitado el aprendizaje y auto-aprendizaje, el uso de tecnologías y la transferencia a problemas y situaciones concretas. Asimismo hemos gestionado conceptualizaciones y sistematizaciones para emprender usos, aplicaciones e interacciones con diversas aplicaciones sobre modelizadores 3D, renderizadores, animaciones, fabricación digital y tratamiento de la imagen. Tales avances han sido interpretados y entendidos a través de secuencias y consecuencias observables, en contacto directo con los 
SIGraDi 2016, XX Congreso de la Sociedad Ibero-americana de Gráfica Digital 9-11, November, 2016 - Buenos Aires, Argentina

objetos, junto a los resultados y la alta factibilidad de transferencia.
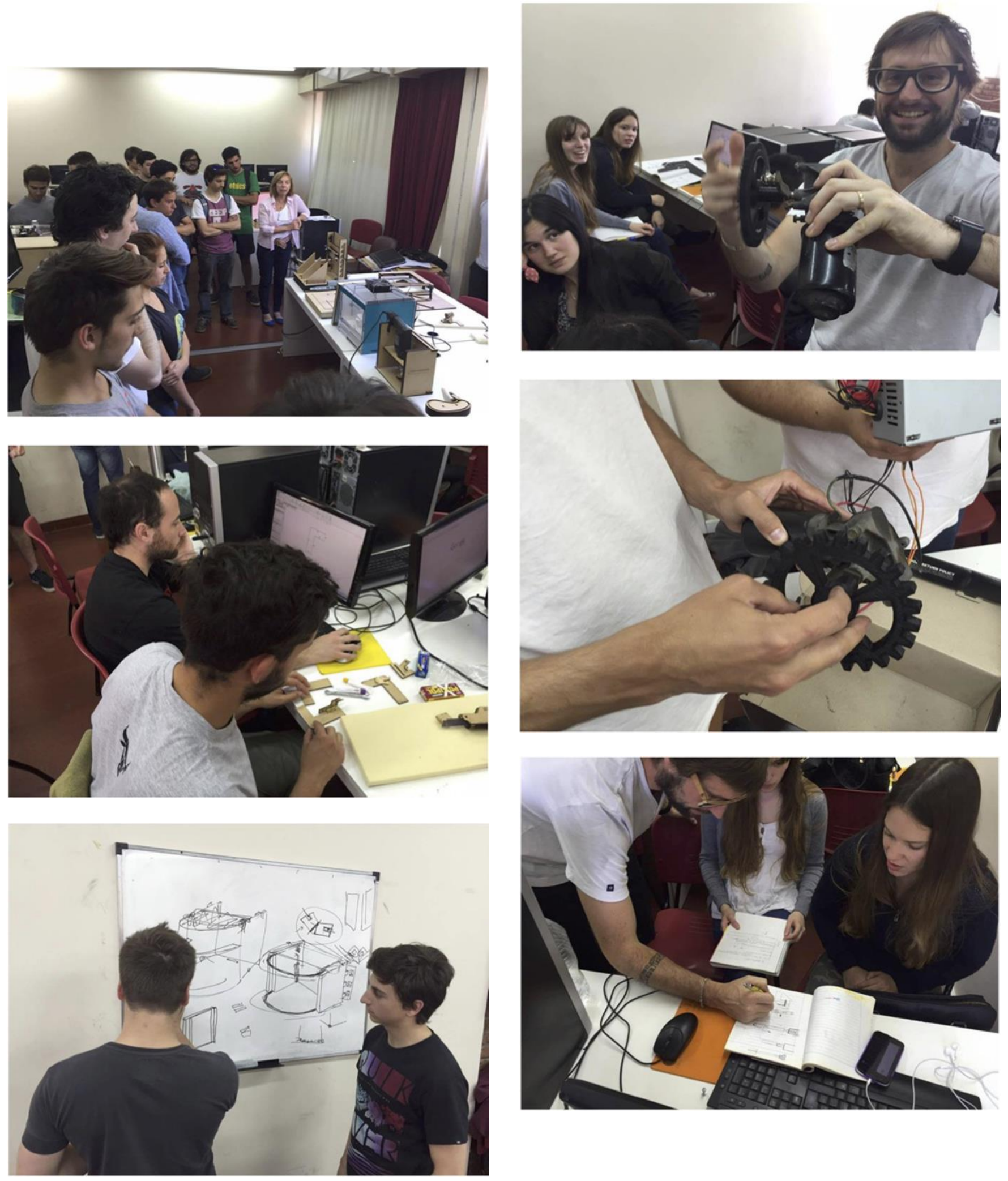


\section{Conclusiones y discusiones}

A manera de reflexiones, consideramos que la experiencia de aprendizaje, en ambientes simulando Fab-Lab, ha generado sentido. Se valoró la naturaleza de los entornos post-digitales como ambientes co-creativos de trabajo, de naturaleza participativa y cooperativa interconectados, para aprovechar oportunidades e interactuar con otros, ampliar los procesos implícitos y enriquecer resultados, así como visualizar y compartir tendencias; la creatividad, para arribar a la capacidad transformadora tendiente a idear, generar, gestionar y producir sistemas ingeniosos; la indagación, para afrontar riesgos y fallas hasta obtener resultados satisfactorios con factibilidad de transferencia hacia situaciones asimilables; los grados de libertad, compromiso y diversión, para desarticular y replantear reglas rígidas y fusionarlas en nuevas normas originales y practicables, afrontar riesgos $y$ fallas hasta obtener resultados satisfactorios.

En términos más amplios ,indagando en la experiencia, hemos comprobado que la emergente Cultura del Hacedor aplicada a la fabricación digital, al software libre y al hardware abierto, despliega una estrategia fundante que produce rupturas con los modelos productivos industriales tradicionales al independizar diseños y medios de producción.

Estimula la creación colaborativa y la innovación distribuida, incorpora experimentación, reflexión y crítica sobre su propia práctica y procesos, así como atiende y subsana las tecnologías que sostienen el propio movimiento.

Redefine al modelo de producción de conocimientos, objetos y entornos, en el que los usuarios son productores y consumidores (pro-sumidores) de tecnologías, contenidos, objetos e información generada por esas mismas infraestructuras tecnológicas tanto en espacios digitales como físicos.

Integra comunidades como público recursivo enfocado en procesos emergentes de prototipado de prácticas, objetos, herramientas, técnicas y diseños, que resultan generadores de valor colectivo sustentados por licencias abiertas.

Finalmente, los productos fabricados fueron puestos a disposición de la comunidad local de estudiantes con posibilidad de ser utilizados y compartidos. Durante el actual ciclo lectivo, estamos replicando y refinando la experiencia enfocada hacia intervenciones de diseño, gestión y comunicación de modelos 3D realísticos en entornos postdigitales, junto a intervenciones exploratorias de prototipado rápido de impresión $3 \mathrm{D}$ de los productos diseñados.

\section{Agradecimientos}

El presente escrito se encuadra en estudios del proyecto 15/B277 SCTyC UNMdP 2014-2015, actualmente continuado en el proyecto 15/B310 SCTyC UNMdP 2016-2017, Grupo EMIDA (CEAC) CIPADI FAUD, ambos con dirección de Diana Rodríguez Barros.

El equipo docente ha estado integrado por los diseñadores industriales Pablo Pellizzoni e Iván Nieto, junto a asistencia de los ayudantes adscriptos Guido Rumitti, Nicolás Ramella, Maximilano Carosella, Lucas Turkalj y Agustín Ponce.

\section{Referencias bibliográficas citadas}

Acaso, M. (2016). ¿Por qué Howard Gardner en vez de Giner? O la necesidad de abordar el cambio metodológico desde pedagogías de proximidad. En María Acaso. Disponible http://tinyurl.com/hcmgs3r

Arango Sarmiento, S. (2016). Maker Movement, una nueva cultura de invención e innovación. En youngmarketing.go Disponible http://www.youngmarketing.co/la-cultura-del-makermovement-y-como-esta-cambiando-el-mundo/

Brown, T. (2016). The Next Big Thing in Design. En Design Thinking. http://designthinking.ideo.com/?p=1451

Disponible

Christensen, C.; Dyer, J.; Gregersen, H. (2011). The innovator's DNA. Boston Ma.: Harvard Business Review Press.

Cobo, C. y Moravec, J. (2011). Aprendizaje invisible. Hacia una nueva ecología de la educación. Barcelona: Edicions Universitat de Barcelona.

Diller, S., Shedroff, N., Rhea, D. (2008). Making Meaning. Berkeley CA: New Riders Press.

Freire, J. (2012). Ecosistemas de aprendizaje, emprendizaje e innovación. En nómada. Disponible http://tinyurl.com/p9rarew (consultado julio 2015)

Llop, P. ( 2013). Cultura libre y peer production: La era maker. En eldiario.es. Disponible http://www.eldiario.es/colaboratorio/Cultura-librepeer-production-maker_6_163843622.html

Rodríguez Barros, D. (2014). Prácticas docentes e interacciones lúdicas en entorno postdigital. En Libro Ponencias $1^{\circ}$ Congreso Latinoamericano Diseño DISUR. San Juan. FAUD UNSJ.

- (2013). Prácticas disruptivas e intervenciones de diseño desde lo lúdico y participativo. En Bernal, M. y Gomez, P. edits. Libro Ponencias Knowledge based Design XVII Congreso SIGRADI. Valparaiso: UTFSM \& SIGRADI. Pp. 533-536. 
SIGraDi 2016, XX Congreso de la Sociedad Ibero-americana de Gráfica Digital 9-11, November, 2016 - Buenos Aires, Argentina 